Sueni de Souza Arouca

\title{
Método implícito para reconstrução de curvas a partir de pontos esparsos
}

Dissertação apresentada como requisito parcial para obtenção do grau de Mestre pelo Programa de Pós-graduação em Matemática Aplicada do Departamento de Matemática da PUC-Rio

Orientador : Prof. Hélio Côrtes Vieira Lopes Co-Orientador: Prof. Luiz Carlos Pacheco R. Velho 


\title{
Sueni de Souza Arouca
}

\section{Método implícito para reconstrução de curvas a partir de pontos esparsos}

Dissertação apresentada como requisito parcial para obtenção do grau de Mestre pelo Programa de Pós-graduação em Matemática Aplicada do Departamento de Matemática do Centro Técnico Científico da PUC-Rio. Aprovada pela Comissão Examinadora abaixo assinada.

\author{
Prof. Hélio Côrtes Vieira Lopes \\ Orientador \\ Departamento de Matemática - PUC-Rio \\ Prof. Luiz Carlos Pacheco R. Velho \\ Co-Orientador \\ IMPA
}

Prof. Marco Antônio Grivet Mattoso Maia

CETUC - PUC-Rio

Prof. Geovan Tavares dos Santos Departamento de Matemática — PUC-Rio

Prof. José Eugenio Leal

Coordenador Setorial do Centro Técnico Científico - PUC-Rio 
Todos os direitos reservados. É proibida a reprodução total ou parcial do trabalho sem autorização da universidade, do autor e do orientador.

\section{Sueni de Souza Arouca}

Graduou-se em Licenciatura em Matemática na Universidade do Estado do Rio de Janeiro - UERJ (Rio de Janeiro, Brasil).

Ficha Catalográfica

Arouca, Sueni de Souza

Método implícito para reconstrução de curvas a partir de pontos esparsos / Sueni de Souza Arouca; orientador: Hélio Côrtes Vieira Lopes; co-orientador: Luiz Carlos Pacheco R. Velho. - Rio de Janeiro : PUC-Rio, Departamento de Matemática, 2006.

v., 66 f: il. ; $29,7 \mathrm{~cm}$

1. Dissertação (mestrado) - Pontifícia Universidade Católica do Rio de Janeiro, Departamento de Matemática.

Inclui referências bibliográficas.

1. Matemática - Tese. 2. Aproximações de curvas implícitas. 3. Modelagem geométrica. 4. MPU. 5. Ridge regression. I. Lopes, Hélio Côrtes Vieira. II. Velho, Luiz Carlos Pacheco R.. III. Pontifícia Universidade Católica do Rio de Janeiro. Departamento de Matemática. IV. Título. 


\section{Agradecimentos}

À minha família pelo apoio e incentivo.

Ao meu orientador Professor Hélio Lopes pelo apoio, carisma de sempre, e incentivo para a realização deste trabalho.

Ao meu namorado Alexandre Fernandes pelo carinho e apoio.

À Capes pelos auxílios concedidos.

Aos funcionários do departamento de Matemática, em especial a Creuza.

Aos meus colegas e amigos da PUC-Rio, em especial aos amigos Marcos Lage e Catiúscia Borges. 


\section{Resumo}

Arouca, Sueni de Souza; Lopes, Hélio Côrtes Vieira; Velho, Luiz Carlos Pacheco R.. Método implícito para reconstrução de curvas a partir de pontos esparsos. Rio de Janeiro, 2006. 66p. Dissertação de Mestrado - Departamento de Matemática, Pontifícia Universidade Católica do Rio de Janeiro.

Nas aplicações em computação gráfica e processamento de imagens, curvas e superfícies implícitas têm sido reconhecidas como a representação mais útil de objetos $2 \mathrm{D}$ ou 3D, principalmente porque elas permitem a descrição de formas complexas por uma fórmula. A maioria dos métodos implícitos usam curvas algébricas para aproximar globalmente a fronteira do objeto em uma imagem binária. Quando a forma do objeto é complexa, é comum elevar o grau da curva a fim de obter mais precisão na aproximação. Uma solução alternativa é decompor hierarquicamente o domínio em partes compactas e obter aproximações locais para o objeto em cada parte, e então juntar os pedaços com o objetivo de obter uma descrição global do objeto. O principal objetivo deste trabalho é apresentar um novo método de aproximação de curvas implícitas a partir de pontos esparsos que melhora o estado da arte.

\section{Palavras-chave}

Aproximações de curvas implícitas. Modelagem geométrica. MPU. Ridge regression. 


\section{Abstract}

Arouca, Sueni de Souza; Lopes, Hélio Côrtes Vieira; Velho, Luiz Carlos Pacheco R.. Implicit method for curve reconstruction from sparse points. Rio de Janeiro, 2006. 66 p. MsC Thesis Department of Matemática, Pontifícia Universidade Católica do Rio de Janeiro.

In the field of computer vision and image analysis, implicit curves and surfaces have been recognized as the most useful representation for $2 \mathrm{D}$ or 3D objects, mainly because they allow description of shapes by a formula. Most of implicit methods uses algebraic curves to fit globally the frontier of the foreground in a binary image. When the foreground shape is complex, it is common to elevate the curve degree in order to obtain more precision on the approximation. An alternative solution is to decompose the domain hierarchicaly in compact parts and obtain local approximation for the object in each part, and then patch all together in order to obtain a global description of the object. The main objective of this work is to present a new method for implicit curve fitting from sparse point that improves the state of the art.

\section{Keywords}

Implicit curve fitting. Geometric modeling. Multi-level partition of unity. Ridge regression. 


\section{Sumário}

\begin{tabular}{lll}
\hline 1 & Introdução & $\mathbf{1 1}$
\end{tabular}

\begin{tabular}{lll}
\hline 1.1 & Descrição do problema & 12
\end{tabular}

$\begin{array}{lll}1.2 & \text { Contribuição } & 13\end{array}$

\begin{tabular}{lll}
\hline 1.3 & Organização da dissertação & 13
\end{tabular}

2 Aproximação por curvas implícitas e partição da unidade $\quad 14$

$\begin{array}{lll}2.1 & \text { Curvas Algébricas } & 14\end{array}$

2.2 Aproximações algébricas por mínimos quadrados 15

$\begin{array}{lll}2.3 & \text { Partição da Unidade } & 19\end{array}$

2.4 O método MPU 20

3 O novo método implícito 25

\begin{tabular}{lll}
\hline 3.1 & Descrição & 25
\end{tabular}

3.2 Implementação 27

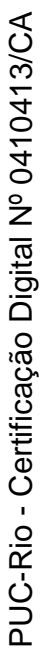

\begin{tabular}{|lll}
4 & Resultados & 34
\end{tabular}

4.1 Curvas Suaves $\quad 35$

4.2 Curvas com singularidades 51

$\begin{array}{lll}4.3 & \text { Completando buracos } & 59\end{array}$

$\begin{array}{lll}5 & \text { Conclusão e trabalhos futuros } & 62\end{array}$

5.1 Trabalhos Futuros 62 


\section{Lista de figuras}

1.1 Dados de entrada: pontos amostrados de uma curva planar e os vetores normais em cada um desses pontos.

1.2 Resultado do método: uma curva implícita que aproxima os dados de entrada da figura 1.1 .

2.1 As figuras (a) e (d) ilustram aproximações obtidas usando o método classical least squares. Já nas figuras (b) e (e) foi utilizado o método gradient one fitting, e nas figuras (c) e (f) o método ridge regression. As linhas pontilhadas são os dados de entrada e as linhas cheias são as aproximações obtidas. O grau do polinômio foi escolhido como sendo 6 para o alicate e 8 para o avião.

2.2 Exemplo da construção de uma Quad-Tree gerando uma subdivisão do domínio adaptada aos pontos.

2.3 Exemplo da região de suporte para cada nó da Quad-Tree.

2.4 Aumentando o raio do círculo do suporte para obter uma garantia de existir solução para o cálculo das quádricas.

2.5 Toro: Figura gerada pelo software MPU.

2.6 Bunny: Figura gerada pelo software MPU.

3.1 Refinamento local que permite uma melhor aproximação global. 28

3.2 Organização da classe Quad_Tree. 29

3.3 Classe Curva_Implícita.

4.1 Dois círculos: método 1 usando um polinômio de grau $d$ com $\mu=0.125$.

4.2 Dois círculos: método 2 usando um polinômio de grau $d$ com $\mu=0.125$ e $\kappa=0.001$.

4.3 Dois círculos: método 3 usando localmente um polinômio de grau $d$ com $\mu=0.125$ e uma Quad-Tree com nível máximo igual a $l_{\max }$.

4.4 Dois círculos: método 4 usando localmente um polinômio de grau $d$ com $\mu=0.125, \kappa=0.001$ e uma Quad-Tree com nível máximo igual a $l_{\max }$.

4.5 Taubin: método 1 usando um polinômio de grau $d$ com $\mu=0.125$.

4.6 Taubin: método 2 usando um polinômio de grau $d$ com $\mu=0.125$ e $\kappa=0.001$.

4.7 Taubin: método 3 usando localmente um polinômio de grau $d$ com $\mu=0.125$ e uma Quad-Tree com nível máximo igual a $l_{\max }$.

4.8 Taubin: método 4 usando localmente um polinômio de grau $d$ com $\mu=0.125, \kappa=0.001$ e uma Quad-Tree com nível máximo igual a $l_{\max }$.

4.9 Sorriso: método 1 usando um polinômio de grau $d$ com $\mu=0.125$.

4.10 Sorriso: método 2 usando um polinômio de grau $d$ com $\mu=0.125$ e $\kappa=0.001$.

4.11 Sorriso: método 3 usando localmente um polinômio de grau $d$ com $\mu=0.125$ e uma Quad-Tree com nível máximo igual a $l_{\max }$. 
4.12 Sorriso: método 4 usando localmente um polinômio de grau $d$ com $\mu=0.125, \kappa=0.001$ e uma Quad-Tree com nível máximo igual a $l_{\text {max }}$

4.13 Hipociclóide: método 1 usando um polinômio de grau $d$ com $\mu=0.125$.

4.14 Hipociclóide: método 2 usando um polinômio de grau $d$ com $\mu=0.125$ e $\kappa=0.001$.

4.15 Hipociclóide: método 3 usando localmente um polinômio de grau $d \operatorname{com} \mu=0.125$ e uma Quad-Tree com nível máximo igual a $l_{\max }$.

4.16 Hipociclóide: método 4 usando localmente um polinômio de grau $d$ com $\mu=0.125, \kappa=0.001$ e uma Quad-Tree com nível máximo igual a $l_{\max }$.

4.17 Cúspide: método 1 usando um polinômio de grau $d$ com $\mu=0.125$.

4.18 Cúspide: método 2 usando um polinômio de grau $d$ com $\mu=0.125$ e $\kappa=0.001$.

4.19 Cúspide: método 3 usando localmente um polinômio de grau $d$ com $\mu=0.125$ e uma Quad-Tree com nível máximo igual a $l_{\max }$.

4.20 Cúspide: método 4 usando localmente um polinômio de grau $d$ com $\mu=0.125, \kappa=0.001$ e uma Quad-Tree com nível máximo igual a $l_{\text {max }}$.

4.21 Nephroid: método 1 usando um polinômio de grau $d$ com $\mu=0.125 .55$

4.22 Nephroid: método 2 usando um polinômio de grau $d \operatorname{com} \mu=0.125$ e $\kappa=0.001$.

4.23 Nephroid: método 3 usando localmente um polinômio de grau $d$ com $\mu=0.125$ e uma Quad-Tree com nível máximo igual a $l_{\max }$.

4.24 Nephroid: método 4 usando localmente um polinômio de grau $d$ com $\mu=0.125, \kappa=0.001$ e uma Quad-Tree com nível máximo igual a $l_{\max }$.

4.25 Círculo: (a) método 3 e (b) método 4 usando localmente um polinômio de grau $d=2 \mathrm{com} \mu=0.17, \kappa=0.01$ e uma Quad-Tree com nível máximo igual a 5

4.26 Sorriso: (a) método 3 e (b) método 4 usando localmente um polinômio de grau $d=2$ com $\mu=0.17, \kappa=0.01$ e uma Quad-Tree com nível máximo igual a 5

4.27 Taubin: (a) método 3 e (b) método 4 usando localmente um polinômio de grau $d=2 \operatorname{com} \mu=0.17, \kappa=0.01$ e uma Quad-Tree com nível máximo igual a 4 


\section{Lista de tabelas}

2.1 Tabela do número de coeficientes do polinômio 15

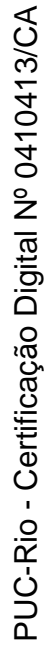

